\title{
PRINCIPALES VARIABLES QUE INCIDEN EN LA INTEGRACIÓN DE LAS CONSTRUCCIONES AGRARIAS EN EL PAISAJE
}

\author{
(PRINCIPAL VARIABLES INFLUENCING THE INTEGRATION OF AGRARIAN \\ CONSTRUCTIONS IN THE LANDSCAPE)
}

Ignacio Cañas Guerrero, Ingeniero Agrónomo ETSE Agrónomos, Universidad de Santiago de Compostela

Ana Isabel García García, Ingeniera Agrónomo ETSI Agrónomos, Universidad Politécnica de Madrid Fecha de recepción: $21-\mathrm{X}-94$ ESPAÑA

\section{RESUMEN}

En el presente artículo se analizan cuáles son las principales variables que intervienen en la integración de las construcciones agrarias en el paisaje. Para ello se realizó un estudio bibliográfico, un estudio sobre el terreno y' un estudio con fotografias aéreas oblicuas.

Como resultado del trabajo se ha obtenido una lista de chequeo de las principales variables que intervienen en el impacto visual de las construcciones agrarias.

\section{SUMMARY}

In present paper would be analyzed wich are the most important variables that have influence on landscape integration of agricultural constructions. A bibliographic study, an "in situ" study and an oblique aerial photography study have been carried out for this.

Check list of the most important variables that take part on visual impact of agricultural construction bave been obtained as a result of this research work.

\section{Introducción}

A la hora de valorar el impacto de las construcciones agrarias en el paisaje cabe preguntarse ¿cuáles son las principales variables que intervienen en la integración de la construcción agraria en el paisaje? Para ello pensamos que es muy útil el disponer de una lista de chequeo que es lo que nos proponemos en el presente trabajo. Para confeccionarla se ha realizado un estudio orientado en dos direcciones:

- 1. Estudio bibliográfico en el que en una primera fase se analizan las publicaciones extranjeras y en una segunda se hace el estudio de la norma- tiva española y, más concretamente, de la normativa que poseen los municipios de la Comunidad de Madrid.

- 2. Estudio sobre el terreno, que se ha realizado en dos etapas:

a) Recorrido piloto por Castilla y León, Asturias y Santander y por los principales municipios de Madrid.

b) Estudio de fotografías aéreas de los municipios rurales. 


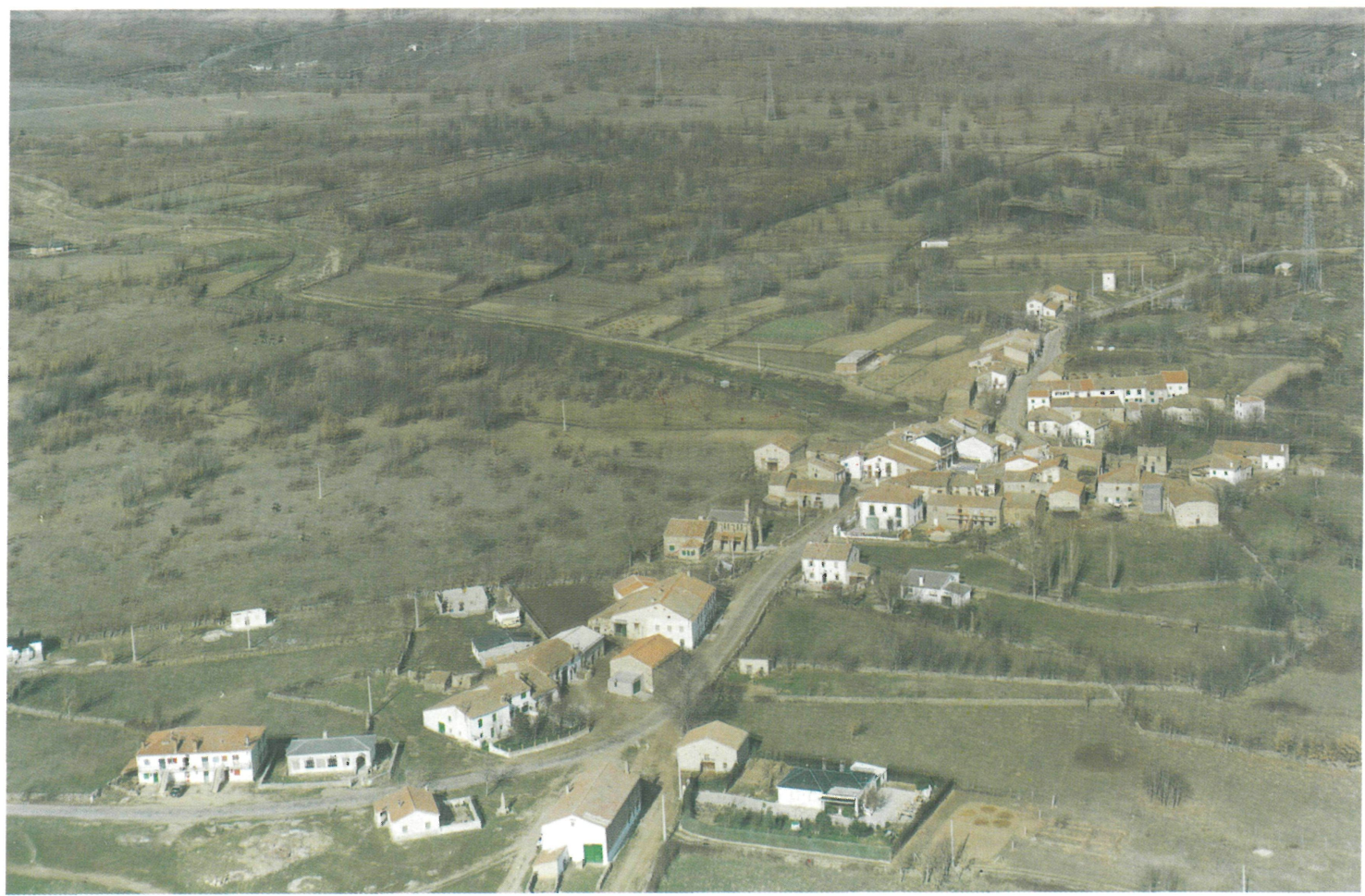

Hercajo de la sierra, Hadrid.

La utilidad que presentan las fotografías aéreas oblicuas para el estudio es grande, ya que resumen en una sola imagen lo que desde el terreno podría significar bastantes tomas fotográficas. En el análisis visual de estas fotografías observamos entre otros: $1 .^{\circ}$ Parece muy importante el color de la cubierta, destacando mucho los cambios. $2 .^{\circ}$ El color de la fachada unido al de la cubierta establece una analogía cromática con el paisaje. $3 .^{\circ} \mathrm{La}$ inclinación de la cubierta y la forma de dos aguas parece que es un factor importante en algunos municipios. $4 .^{\circ}$ El color de la carpintería puede tener un efecto negativo si son colores muy saturados (fotografía facilitada por FOAT).

Con los dos estudios bibliográfico y sobre el terreno pretendemos elaborar una lista de chequeo de las diversas variables que intervienen en la integración de las construcciones en el paisaje. En términos generales hablaremos de instalación, entendiendo por tal a las construcciones aisladas o grupos con una actividad común.

\section{Variables señaladas en la bibliografia internacional}

De las diversas publicaciones extranjeras que tratan del impacto de las construcciones agrarias, algunas se centran más en la pérdida de las tipologías tradicionales (Vonholdt, K., 1982; Damm, 1988). Otras exponen la preocupación por la proliferación de las nuevas construcciones (Chiappini, 1988). Sólo un pequeño porcentaje se centra en los parámetros que inciden en la integración, no coincidiendo todos los autores en señalar los mismos.

Entre las variables que los diversos estudios consideran que influyen en la integración de una cierta construcción en el paisaje tenemos:
- La localización o situación de los edificios en el terreno, la oriențación de éstos, el tamaño, forma, color, textura y materiales característicos de la instalación.

- Algunos autores exponen la importancia de los detalles, acabados, cerramientos o acondicionamiento paisajístico, otros señalan el efecto de ciertos elementos adyacentes como son: la vegetación, movimiento de tierras o la pavimentación. A veces se destaca la importancia de ciertas partes del edificio para la integración como son: paredes, aleros, chimeneas, entradas, etc. En concreto, se puede hacer la siguiente enumeración clasificada por autores:

- Penfold (1979): La localización. El tamaño. La apariencia (composición de formas). El color y la textura. Los detalles.

- O’Farrell, F. (1987): La localización. Entradas. Forma. Escala. Color. Vegetación. Materiales. Cerramientos. Detalles. 
- Cull (1987): Los edificios tradicionales. La localización. La forma y escala. El color. Los materiales. Los detalles. El acondicionamiento paisajístico.

- Geoghegan, P. y Culligan, D. (1988): La localización. Vegetación. Movimiento de tierras. Protección del viento. Orientación. Forma. Detalles. Escala. Materiales y tratamiento. Entradas. Urbanización. Paredes. Aleros. Cubiertas. Chimeneas. Entradas y porches. Puertas. Ventanas. Tamaño.

- Dolby et al. (1988): La localización. La forma. Los materiales. El color. Los detalles.

- Shakespeare, R. P. y Dodd, V. A. (1989): La localización. El acondicionamiento paisajístico. El color. La forma. El tamaño.

- Di Facio, J. (1989): La localización. La forma. Los materiales. El color. La textura. Acondicionamiento paisajístico: la pavimentación, la vegetación, los elementos de separación, los detalles.

Entre éstos tiene especial relevancia la publicación de Cull (1987), ya que es un resumen de las principales guías de diseño del Reino Unido al incluir las siguientes: Peak National Park, Broads, North Bedforshire Borough Council, Cheshire County Council, East Lindsey District Council, Cambridgeshire County Council, East Herts District Council, Stroud District Council, Exmoor National Park y Dartmoor National Park.

En la relación anterior se puede observar que existen unos elementos comunes como son: la localización, la forma, la escala, el color, los materiales y los detalles.

En la localización suelen especificar tanto la orientación como la ubicación de la instalación; la importancia de la localización estriba en que nos determina desde dónde va a ser vista la construcción. En este sentido suelen recomendar el evitar la colocación de la instalación en colinas o en zonas donde tapen la línea del horizonte $y$, en general, las zonas donde sean observadas por un gran número de visitantes.

Dentro de la localización también se hace referencia a la situación con relación a otros edificios aconsejándose las instalaciones juntas antes que las dispersas (Cull, 1987).

Por lo que se refiere a la forma y a la escala de las nuevas edificaciones, diversas publicaciones las sue-

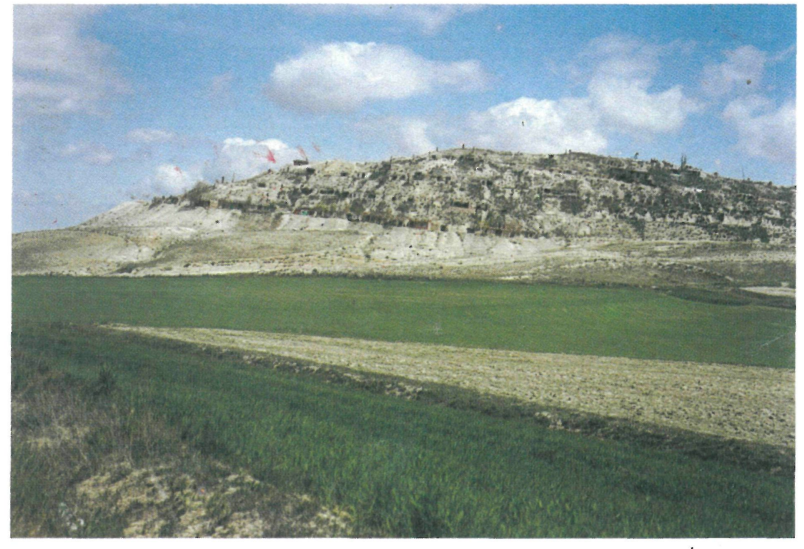

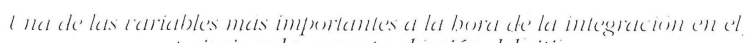
paisaje es la correcla elección del silio.

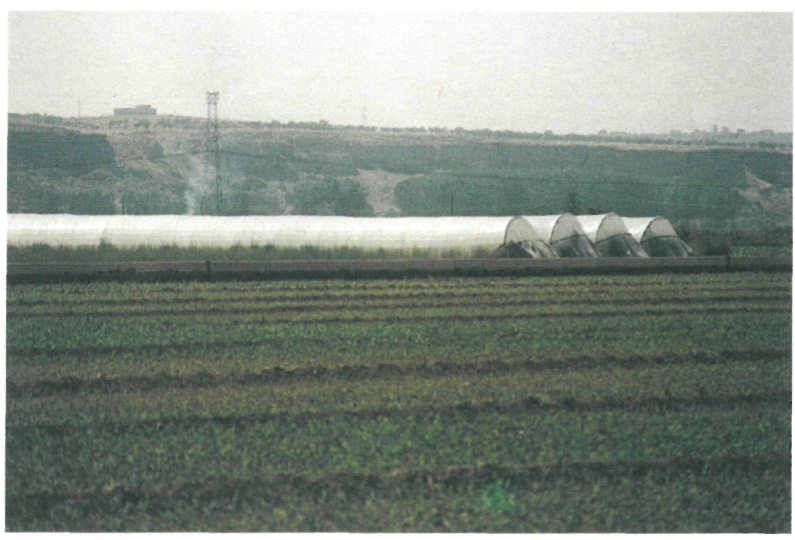

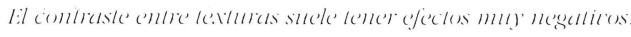

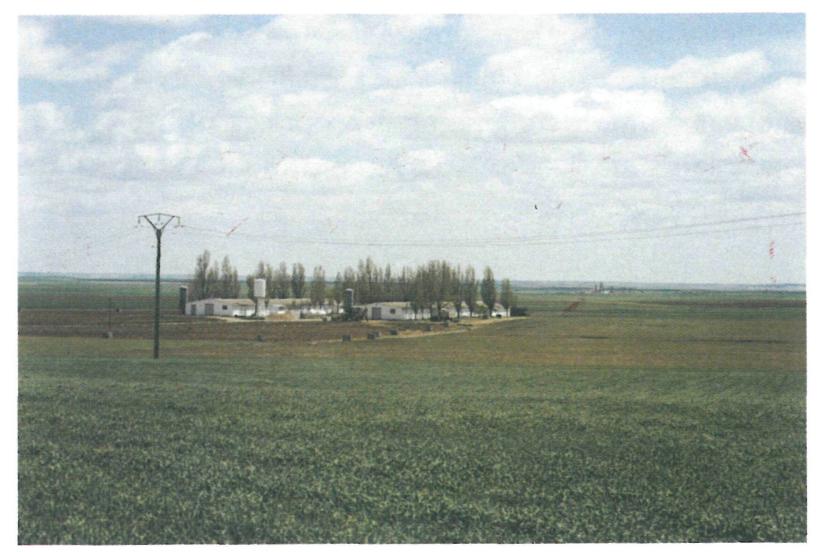

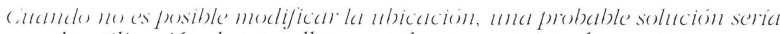
la utilización de pantallas vegetales que suavicen los contornos. 


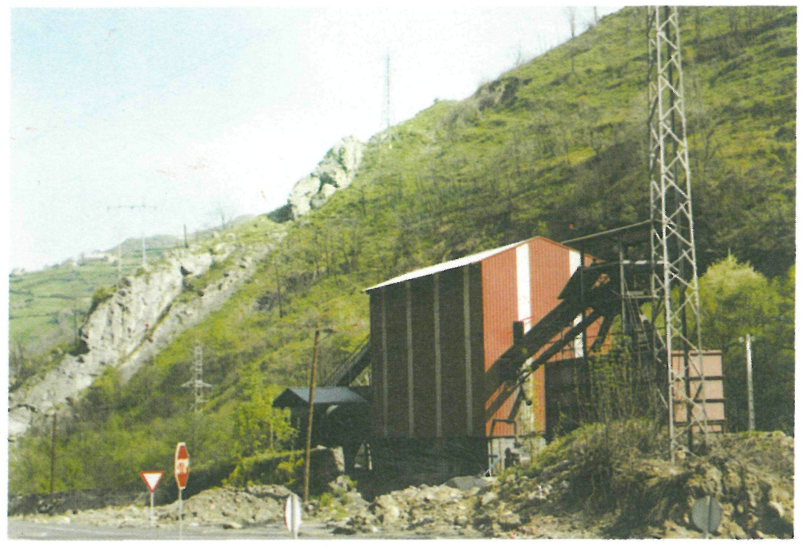

Los colores muy' saturados suelen dar problemas.

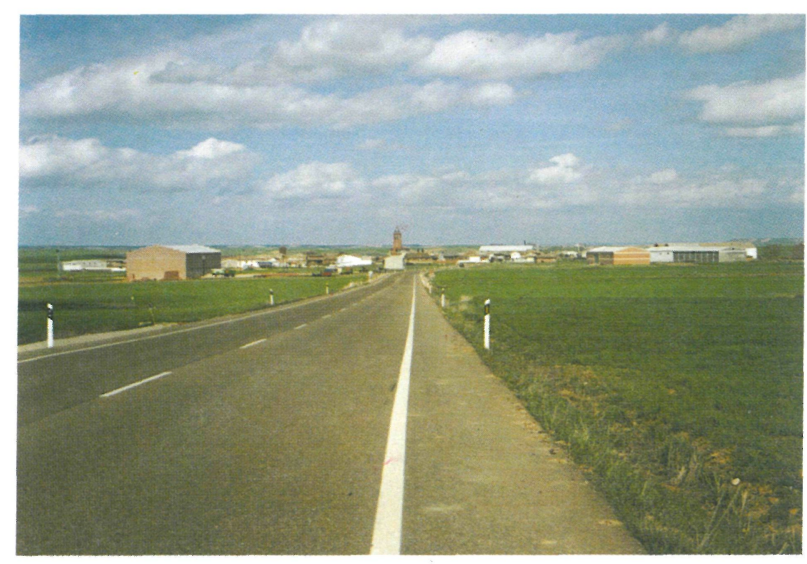

En entrada y salida de los núcleos rurales suelen establecerse las diversas industrias o naves, rompiendo el perfil del núcleo y restando importancia a las demás construcciones. len considerar, ya que generalmente son muy distintas a las tradicionales; muchas veces los nuevos edificios necesitan longitudes de más de $50 \mathrm{~m}$, además de que estas variaciones en formas y tamaños o escala suelen verse agravadas por la utilización de materiales artificiales que en algunos casos contrastan con el entorno.

En cuanto al color se suele recomendar que las cubiertas sean más oscuras que las paredes (Design Council, 1974; Di Facio, 1989; Shakespeare, R.P. y Dodd, V. A., 1989), también se suele indicar la importancia del color para la integración, ya que a veces se consigue que el edificio no aparezca de un gran tamaño (por ejemplo, con la utilización de varios colores en la fachada).
Con relación a los materiales, algunas veces se estudia como textura (Penfold, 1979), en otros aparecen reflejados los materiales a usar en diversos elementos (Geoghegan, P. y Culligan, D., 1988; Knowles, P. et al., 1977). Hay también algunas publicaciones que se centran más en las características de precio y características físicas de los materiales a emplear. En el estudio de los materiales intervienen otras variables como son los costos, mantenimiento, etc., que condicionan muchas veces los aspectos estéticos; no obstante, como señala Di Facio (1989), el uso de diversos materiales en la fachada puede ayudar a romper la uniformidad de las superficies, disminuyendo el efecto escala.

Por lo que se refiere a los detalles no todos citan los mismos; así, entre otros, tenemos: los aleros (Damm, 1982), las bajantes y los canalones (Dolby et al., 1988), puertas y huecos de ventilación (Broads, 1985; Dolby et al., 1988).

\section{Las variables señaladas en las Normas Subsidiarias de la Comunidad Autónoma de Madrid}

Esto ya se desarrolló en otro número de esta revista (ver "Informes de la Construcción", número 429, páginas 33 y siguientes); en síntesis, se estudiaron 20 municipios de los que se analizaron 20 variables, estableciéndose una clasificación por orden de importancia de los diversos parámetros.

1. Altura del edificio (17 municipios).

2. Dimensiones de cuerpos y elementos salientes (16 municipios).

3. Inclinación de las cubiertas (15 municipios).

4. Altura de los cerramientos (13 municipios).

4. Dimensiones de los aleros (13 municipios).

4. Forma y dimensiones de la carpintería (13 municipios).

5. Materiales de la fachada (12 municipios).

6. Materiales de la cubierta (10 municipios).

7. Color de la cubierta ( 9 municipios).

7. Color de la fachada (9 municipios).

7. Materiales del cerramiento (9 municipios).

8. Textura de elementos exteriores (7 municipios).

9. Color del cerramiento ( 5 municipios).

10. Dimensiones de huecos de puerta (4 municipios). 
10. Materiales de carpintería (4 municipios).

10. Color de la carpintería (4 municipios).

11. Dimensiones hueco ventanas (2 municipios).

12. Textura carpintería (1 municipio).

12. Impostas o molduras (1 municipio).

12. Persianas (1 municipio).

Por estas observaciones pensamos que los elementos estéticos más importantes en las Normas son los siete siguientes (numerados por orden de importancia):

1. Altura del edificio.

2. Inclinación de la cubierta.

3. Altura de los cerramientos.

4. Materiales de la fachada.

5. Materiales de la cubierta.

6. Color de la fachada.

7. Color de la cubierta.

Además de los condicionantes estéticos, ya enunciados, las Normas Subsidiarias actuales intentan proteger el patrimonio paisajístico fijando las siguientes normas:

- Normas de protección del perfil del núcleo, en el que se intenta proteger el casco antiguo y el borde perimetral del núcleo.

- Normas que intentan conservar lo que llaman "estructura paisajística tradicional" que incluyen:

a) Protección de la topografía, impidiendo actuaciones que alteren las características morfológicas del terreno.

b) Protección de cauces naturales y del arbolado correspondiente, así como de acequias y canales de riego.

c) Protección de plantaciones y masas forestales.

d) Protección de caminos de acceso, cañadas, veredas, etc.

e) Protección de construcciones, tales como: sistemas de cercas, corrales, apriscos, casetas de aperos de labranza, etc., que configuren la bondad del paisaje.
- Normas que pretenden proteger las vistas, lo que suelen llamar visualizaciones. En concreto teniendo en cuenta tres supuestos diferenciados:

1. Visualizaciones del entorno desde el casco urbano.

2. Visualizaciones del casco desde el entorno.

3. Visualizaciones interiores del casco.

- Normas sobre los anuncios o publicidad exterior.

- Normas sobre la plantación de arbolado, señalando la necesidad de plantar dos filas de árboles de especies propias del entorno (salvo que el análisis paisajístico y ecológico aconseje otra solución).

- Obligatoriedad de los estudios de impacto ambiental.

\section{Estudio sobre el terreno: estudio piloto}

El estudio piloto se realizó con un recorrido por Castilla y León, Asturias y Santander, además de por algunos municipios de Madrid: Titulcia, Torrelaguna, Patones, Montejo de la Sierra, El Escorial, Navalcarnero, Morata de Tajuña, Brunete, Meco y Miraflores de la Sierra.

Durante estas salidas las variables que más llamaron nuestra atención, con relación a los recursos estéticos, fueron: la altura de los edificios, la forma de las cubiertas y el color. La textura cuando se utilizaban materiales brillantes era un factor distorsionador muy importante. Con relación a los condicionantes de posición de la instalación destacaban: interrupción de la línea del horizonte, tapar vistas y la fragmentación del espacio paisajístico.

\section{Estudio a partir de las fotografias aéreas}

Para completar los puntos de vista observados se pidieron las fotografías aéreas oblicuas de los diversos municipios rurales y villas de la Comunidad de Madrid, de éstos se consiguieron 30 municipios: Batres, Belmonte del Tajo, Brunete, Camporreal, Cervera de Buitrago, Cobeña, El Berrueco, El Atazar, Fuentidueña del Tajo, Gascones, Horcajuelo de la Sierra, Horcajo de la Sierra, La Hiruela, La Acebeda, la Serna del Monte, Las Rozas de Puerto Real, Olmeda de las Fuentes, Pezuela de las Torres, 
TABLA 1. Lista de chequeo de las principales variables que intervienen en el impacto visual de las construcciones agrarias

\begin{tabular}{|c|c|c|c|}
\hline ASPECTOS GENERALES & 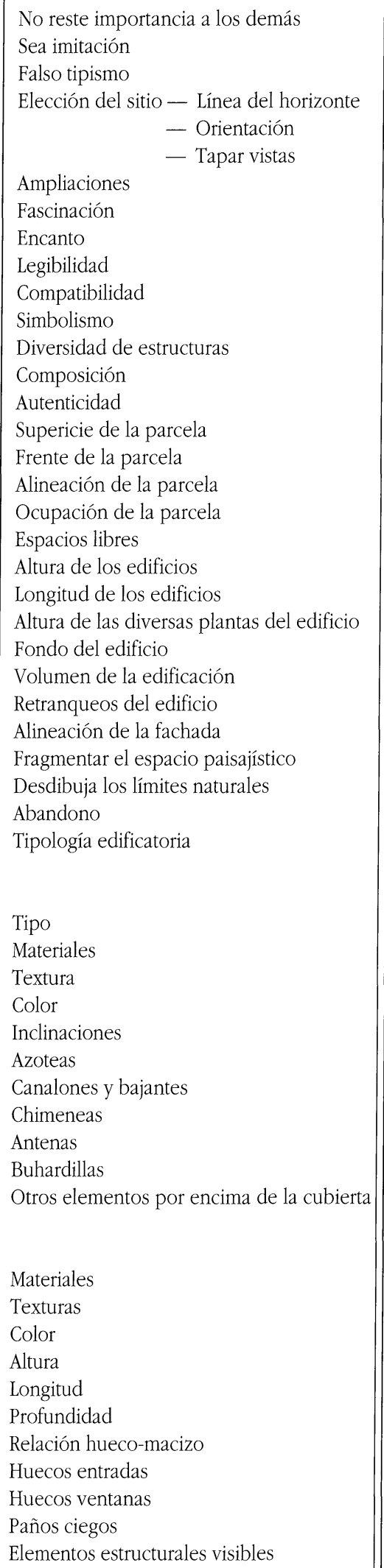 & $\begin{array}{l}\text { CUERPOS SALIENTES } \\
\text { ELEMENTOS ENTRANTES } \\
\text { PATIOS } \\
\text { CERCAS } \\
\text { ACCESOS }\end{array}$ & $\begin{array}{l}\text { Pavimentación } \\
\text { Aparcamiento } \\
\text { Garaje } \\
\text { Almacenes } \\
\text { Silos } \\
\text { Depósitos } \\
\text { Sistemas de iluminación } \\
\text { Maquinaria } \\
\text { Tendido eléctrico } \\
\text { Porches } \\
\text { Jardines } \\
\text { Movimientos de tierra } \\
\text { Vertederos, escombros }\end{array}$ \\
\hline
\end{tabular}


Quijorna, Redueña, Robregordo, Somosierra, Titulcia, Torres de la Alameda, Valdepiélagos, Valdetorres del Jarama, Villalbilla, Villanueva de Perales, Villanueva de la Cañada y Villavieja de Lozoya.

La utilidad que presentan las fotografías aéreas oblicuas para el estudio es grande, ya que resumen en una sola imagen lo que desde el terreno podría significar bastantes tomas fotográficas. En el análisis visual de estas fotografías observamos lo siguiente:

1. Parece muy importante el color de la cubierta, destacando mucho los cambios.

2. El color de la fachada unido al de la cubierta establece una analogía cromática con el paisaje.

3. La inclinación de la cubierta y la forma de dos aguas parece que es un factor importante en algunos municipios.
4. El color de la carpintería puede tener un efecto negativo si son colores muy saturados.

5. La altura de los edificios y la agrupación de éstos si ocasiona un volumen grande de edificación continua puede en algunos casos ser importante.

6. En algunos casos el tipo de asentamiento puede ser característico.

\section{Lista de chequeo}

Después de haber examinado, desde el punto de vista bibliográfico y de estudio del terreno, las diversas variables que pueden intervenir en el impacto de las construcciones agrarias, podemos construir una lista de chequeo de éstas como aparece en la Tabla 1.

\section{BIBLIOGRAFIA}

(1). BROADS (1985): Agricultural Buildings, Broads design Guidance leaflet, número 2, Broads Authority.

(2). CAÑAS GUERRERO, I. (1993): Integración de las construcciones agrarias en el paisaje: el color, tesis doctoral, Universidad Politécnica de Madrid. Sin publicar.

(3) CAÑAS GUERRERO, I. (1994): Las construcciones agrarias en la Comunidad de Madrid y sus condicionantes estéticos según las normas subsidiarias, Informes de la Construcción, 1994. volumen 45, número 429, páginas 33-45.

(4) CULL, S. (1986): "Farm Buildings and Planining Control", Farm Buildings and Engineering, volumen 3, número 2.

(5) CULL, S. (1987): "Design Guidance for Farm Buildings", Farm Buildings and Engineering. 3. (3), páginas 12-16.

(6) CULL, S. (1989): "New Planning Controls on Farm Buildings", Farm Buildings \& Engineering. 1 (6), páginas 31-33.

(7) CHIAPPINI, U. (1988): "La progettazione degli edifici agricoli oggi e domani", Genio rurale (LI) 9: 5-9.
(8) DAMM, T. (1979): Umbau alter bauernhäuser in NordrheinWestfalen. Landtechnik, 1. Januar, páginas 18-21.

(9) DAMM, T. (1982): "Landschaftsbezogenes Bauen in Nordrhe Westfalen", Landtechnik, volumen 37 (12) páginas 540-541.

(10) DAMM, T. (1988): Landwirtschaftliches bauen in der Landschaft, Landtechnik 10, volumen 43, páginas 428-430.

(11) DESIGN COUNCIL (1974): Revised Colour Finshes for Factory-Made Cladding Used in Farm Buildings, Design Council.

(12) DI FACIO, J. (1989): "Designing Agricultural Buildings in Relation to the Landscape", 11. International Congress on Agricultural Engineering, Land and Water Use, Dodd \& Grace (eds.) Balkema, volumen 2, páginas 1191-1198.

(13) DIRECCIÓN GENERAL DE URBANISMO (1981): Plan General de Ordenación Urbana del término municipal de Navalcarnero, Consejería de Política Territorial, Comunidad de Madrid, Madrid. 
(14) DIRECCIÓN GENERAL DE URBANISMO (1984): Normas Subsidiarias de Planeamiento del término municipal de Rozas de Puerto Real, Consejería de Política Territorial, Comunidad de Madrid, Madrid.

(15) DIRECCIÓN GENERAL DE URBANISMO (1985a): Normas Subsidiarias de Planeamiento del término municipal de Chinchón, Consejería de Política Territorial, Comunidad de Madrid, Madrid.

(16) DIRECCIÓN GENERAL DE URBANISMO (1985b): Normas Subsidiarias de Planeamiento del término municipal de San Martín de Valdeiglesias, Consejería de Política Territorial, Comunidad de Madrid, Madrid.

(17) DIRECCIÓN GENERAL DE URBANISMO (1986a): Normas Subsidiarias de Planeamiento del término municipal de Gargantilla de Lozoya, Consejería de Política Territorial, Comunidad de Madrid, Madrid.

(18) DIRECCIÓN GENERAL DE URBANISMO (1986b): Normas Subsidiarias de Planeamiento del término municipal de Colmenar de Oreja, Consejería de Política Territorial, Comunidad de Madrid, Madrid.

(19) DIRECCIÓN GENERAL DE URBANISMO (1986c): Normas Subsidiarias de Planeamiento del término municipal de Redueña, Consejería de Política Territorial, Comunidad de Madrid, Madrid.

(20) DIRECCIÓN GENERAL DE URBANISMO (1986d): Normas Subsidiarias de Planeamiento del término municipal de Horcajo de la Sierra, Consejería de Política Territorial, Comunidad de Madrid, Madrid.

(21) DIRECCIÓN GENERAL DE URBANISMO (1986e): Normas Subsidiarias de Planeamiento del término municipal de Gargantilla de Lozoya, Consejería de Política Territorial, Comunidad de Madrid.

(22) DIRECCIÓN GENERAL DE URBANISMO (1987a): Normas Subsidiarias de Planeamiento del término municipal de Batres, Consejería de Política Territorial, Comunidad de Madrid, Madrid.

(23) DIRECCIÓN GENERAL DE URBANISMO (1987b): Normas Subsidiarias de Planeamiento del término municipal de Torrelaguna, Consejería de Política Territorial, Comunidad de Madrid, Madrid.

(24) DIRECCIÓN GENERAL DE URBANISMO (1987c): Normas Subsidiarias de Planeamiento del término municipal de Cobeña, Consejería de Política Territorial, Comunidad de Madrid, Madrid
(25) DIRECCIÓN GENERAL DE URBANISMO (1987d): Normas Subsidiarias de Planeamiento del término municipal de Puebla de la Sierra, Consejería de Política Territorial, Comunidad de Madrid, Madrid.

(26) DIRECCIÓN GENERAL DE URBANISMO (1987e): Normas Subsidiarias de Planeamiento del término municipal de Brea del Tajo, Consejería de Política Territorial, Comunidad de Madrid, Madrid.

(27) DIRECCIÓN GENERAL DE URBANISMO (1987f): Normas Subsidiarias de Planeamiento del término municipal de Ciempozuelos, Consejería de Política Territorial, Comunidad de Madrid, Madrid.

(28) DIRECCIÓN GENERAL DE URBANISMO (1987g): Normas Subsidiarias de Planeamiento del término municipal de Valdetorres del Jarama, Consejería de Política Territorial, Comunidad de Madrid, Madrid

(29) DIRECCIÓN GENERAL DE URBANISMO (1987h): Normas Subsidiarias de Planeamiento del término municipal de Villanueva de la Cañada, Consejería de Política Territorial, Comunidad de Madrid, Madrid

(30) DIRECCIÓN GENERAL DE URBANISMO (1987i): Normas Subsidiarias de Planeamiento del término municipal de Valdemoro, Consejería de Política Territorial, Comunidad de Madrid, Madrid

(31) DIRECCIÓN GENERAL DE URBANISMO (1988): Normas Subsidiarias de Planeamiento del término municipal de Estremera, Consejería de Política Territorial, Comunidad de Madrid, Madrid

(32) DIRECCIÓN GENERAL DE URBANISMO (1989): Normas Subsidiarias de Planeamiento del término municipal de Montejo de la Sierra, Consejería de Política Territorial, Comunidad de Madrid, Madrid.

(33) DIRECCIÓN GENERAL DE URBANISMO (1990a): Normas Subsidiarias de Planeamiento del término municipal de Torrejón de Velasco, Consejería de Política Territorial, Comunidad de Madrid, Madrid.

(34) DIRECCIÓN GENERAL DE URBANISMO (1990b): Normas Subsidiarias de Planeamiento del término municipal de Lozoyuela-Las Navas-Siete Iglesias, Consejería de Política Territorial, Comunidad de Madrid, Madrid.

(35) DIRECCIÓN GENERAL DE URBANISMO (1991a): Normas Subsidiarias de Planeamiento del término municipal de Cenicientos, Consejería de Política Territorial, Comunidad de Madrid, Madrid. 
(36) DIRECCIÓN GENERAL DE URBANISMO (1991b): Normas Subsidiarias de Planeamiento del término municipal de Buitrago de Lozoya, Consejería de Política Territorial, Comunidad de Madrid, Madrid.

(37) DIRECCIÓN GENERAL DE URBANISMO (1991c): Normas Subsidiarias de Planeamiento del término municipal de Chapineria, Consejería de Política Territorial, Comunidad de Madrid, Madrid.

(38) DOLBY, C., et al. (1988): Rural Constructions in Timber, Department of Farm buildings, Lund, Sweden.

(39) GEOGHEGAN, P., and CulligAN, D. (1988): Building Sensitively in the Landscapes of county Wexford, Wexford Co. Council by The Housing and urban Design Research Unit.

(40) KNOWLES, P., et al. (1977): Building Design Guide, Peak National Park.

(41) NAVAjAS, P. (1983a): Arquitectura vernácula de Madrid, Comunidad de Madrid, Consejería de Ordenación del Territorio, Medio Ambiente y Vivienda, Madrid.
(42) NAVAJAS, P. (1983b): Normativa urbanistica y arquitectónica sobre la vivienda rural en la provincia de Madrid, Comunidad de Madrid, Consejería de Ordenación del Territorio, Medio Ambiente y Vivienda, Madrid.

(43) O'FARRELL, F. (1987): Farm Buildings and Environment, Foras Taluntais, Dublín.

(44) PENFOLD, H. G. (1979): "Landscape and Farm Buildings", Adas Quarterly Review (UK), páginas 223-230.

(45) SHAKESPEARE, R. P., and DODD, V. A. (1989): "A Technique for Assessing the Correct Colour Scheme and Associated Landscaping for Buildings in Rural Landscapes", 11. International Congress on Agricultural Engineering, Land and Water Use, Dod \& Grace (eds.), Balkema, páginas 1207-1212.

(46) VONHOLDT, K. (1982a): "Landschaftsbezogene Bauweisen in niedersachsen. Entwicklungsstufen des niederdeutschen hallenhauses", Lanoltechnik, volumen 37 (12), páginas 534539 .

\section{Publicación del Instituto Eduardo Torroja - CSIC}

\section{Número monográfico de INFORMES}

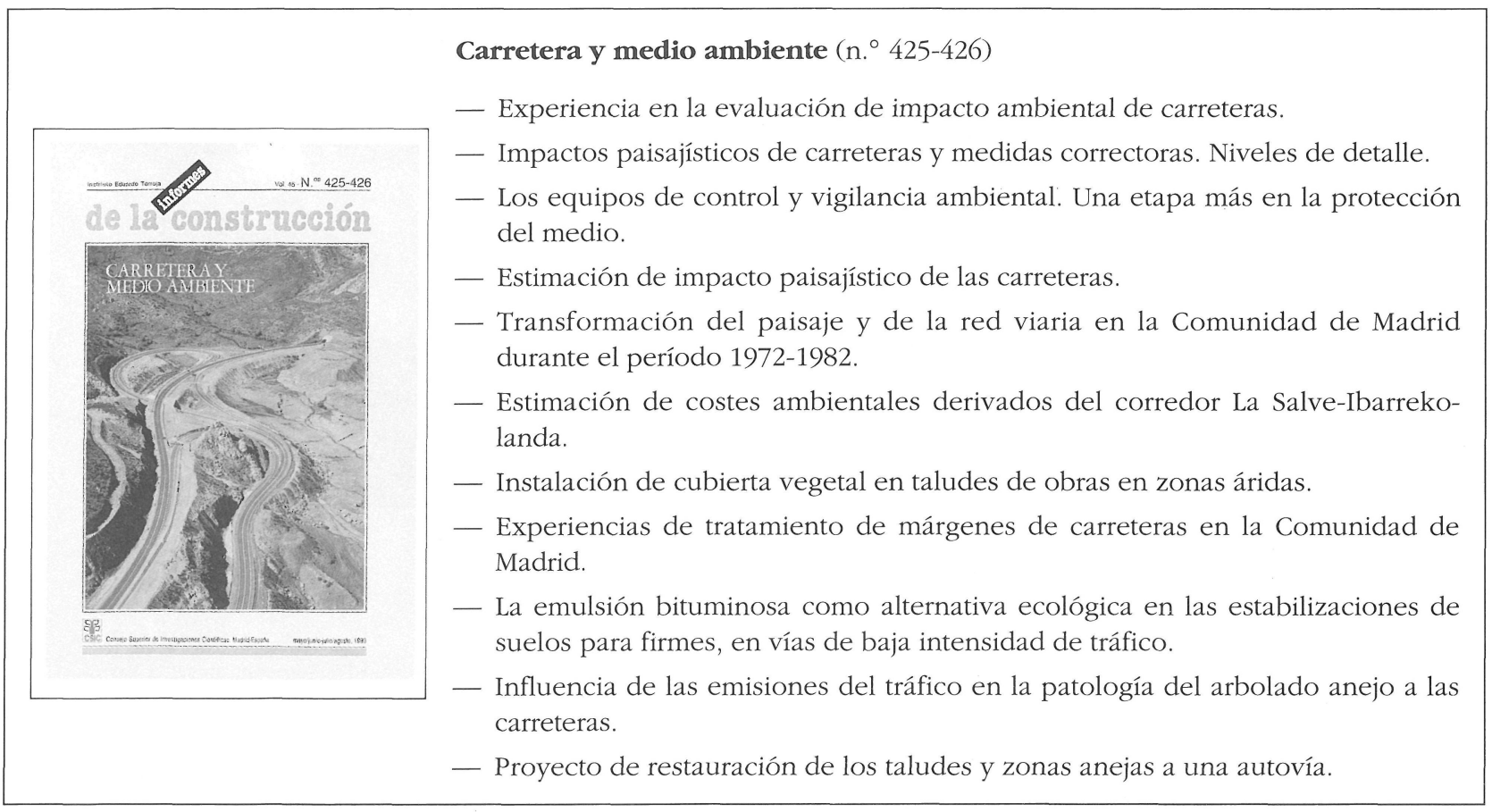

